\title{
Principios educativos ocio humanista en los programas de formación permanente de adultos de las universidades españolas ${ }^{1}$
}

\author{
Leisure humanistic educational principles in lifelong learning \\ programs for adults of Spanish universities
}

\author{
M. a Luisa Amigo Fernández de Arroyabe \\ Yolanda Lázaro Fernández • yolanda.lazaro@deusto.es \\ Iñaki Moreno Navarro \\ DEUSTUKO UNIBERTSITATEA/UNIVERSIDAD DE DEUSTO
}

Recibido: 04/04/2016

Aceptado: 1/07/2016

\section{Resumen}

La formación permanente de adultos es una preocupación de las universidades españolas desde hace treinta años. El objeto de este trabajo se concreta en analizar los principios educativos de las propuestas que las Universidades españolas ofertan a las personas adultas o mayores. La metodología utilizada ha consistido en un análisis de contenido de los documentos sintetizados públicamente en la información mostrada en la página web de cada centro, tomando como marco las universidades de la Asociación Estatal de Programas Universitarios para mayores (AEPUM). Se ha realizado una interpretación hermenéutica de los planteamientos de los Programas Universitarios para Mayores, tratando de leerlos desde la teoría del ocio. Se ha llevado a cabo una revisión bibliográfica de los fundamentos teóricos que sustentan el modelo de formación como ocio y de los estudios existentes sobre los programas de mayores. Los aspectos novedosos de este análisis resaltan que las ofertas formativas de estos programas han tratado de contribuir a la educación a lo largo de la vida de sus estudiantes. El análisis de los principios desde la teoría del ocio apunta a la necesidad de reforzar la importancia de la satisfacción como meta de la formación en los adultos y precisar el concepto de autotelismo para favorecer una formación como ocio.

Palabras clave: Ocio, educación, adultos, programas universitarios.

\section{Abstract}

Adult lifelong learning has been a concern of most Spanish universities for the last thirty years. The purpose of this paper is to analyse some particular educational principles of the proposals that the Spanish Universities offer to adults. The methodology used consists of a content analysis of the documents summarized in the information displayed on the web page of each school, taking as a framework the universities of the State Association of University Programs for the elderly (AEPUM). There has been a hermeneutic interpretation of the approaches of the University Programs for Seniors, the analysis being based on the theory of leisure existent literature. This paper also offers a review of the literature of the theoretical foundations that support leisure studies as a learning model and also of the current studies on programs for Seniors. The novel aspects of this analysis highlight that the training offers of these programs have tried to contribute to the students' education throughout their whole lives. The analysis of the above mentioned principles from the point of view of the theory of leisure points to the need to reinforce the importance of satisfaction as the goal of the training programmes for adults and also to specify the concept of autotelismo in order to promote leisure studies as a training offer.

Keywords: Leisure, education, adults, university programs.

\footnotetext{
1 Este estudio forma parte del Proyecto «Formación para un ocio experiencial valioso a lo largo de la vida: contribución de los itinerarios de ocio al envejecimiento satisfactorio» (ITINERE), financiada en la convocatoria del Plan Nacional de I+D+i (2012), por la Secretaría de Estado de Investigación, Desarrollo e Innovación, adscrita al Ministerio de Economía y Competitividad, con referencia EDU2012-38052.
} 


\section{INTRODUCCIÓN}

En el último tercio del siglo xx, las universidades españolas han sido conscientes de la necesidad de contribuir a la formación a lo largo de la vida y al envejecimiento satisfactorio de los estudiantes mayores. En este trabajo nos interesa profundizar en los principios educativos que son la base de las propuestas que las Universidades españolas ofertan a las personas adultas o mayores. Para abordar este tema, y aun siendo conscientes de las limitaciones de hacerlo en un artículo de esta naturaleza, hemos planteado un enfoque que no se mantuviera en un punto de vista exclusivamente descriptivo. Hemos partido de la cercana relación que los autores mantenemos con la propuesta concreta del Instituto de Estudios de Ocio de la Universidad de Deusto, en el campo de los programas universitarios para mayores. Desde esta experiencia analizamos, en primer lugar, los fundamentos teóricos que sustentan el modelo de formación como ocio, que nutre la ya larga trayectoria del Instituto en investigación sobre ocio humanista y ocio creativo. En segundo lugar, estudiamos desde la base teórica del ocio humanista los programas de otras universidades españolas. Para contextualizar correctamente nuestro acercamiento, comenzamos por unas breves notas acerca del desarrollo de los programas universitarios para mayores vinculados a la Asociación Estatal de Programas Universitarios para Mayores (AEPUM). A continuación, presentamos las bases teóricas que constituyen el modelo del ocio humanista que ha servido de base a los programas del Instituto de Estudios de Ocio haciendo referencia a algunas ideas fundamentales (experiencia, libertad, satisfacción, autotelismo, personalización y valores) y hacemos un breve apunte acerca de cómo tales ideas han dado como fruto los distintos programas para, finalmente, y basándonos en una plantilla constituida a partir los conceptos, comprobar cómo, de manera explícita o implícita, están presentes o no en las propuestas de las universidades estudiadas.

\section{ESTADO DE LA CUESTIÓN DE LOS PROGRAMAS UNIVERSITARIOS PARA MAYORES (PUM) EN ESPAÑA}

La investigación es coincidente en destacar la existencia de tres generaciones de programas socioeducativos para las personas mayores. La primera generación se sitúa en los años 70 del pasado siglo, cuando el debate estaba en la necesidad de desarrollo de distintas modalidades de formación, explicitada como formación no formal e informal y coincidiendo con la creación de la primera Universidad de la Tercera Edad en Toulouse, Francia, en 1973. La segunda generación de programas socioeducativos para personas mayores se desarrolla en la segunda mitad de los setenta cuando desde el ámbito de la gerontología se reconoce la dimensión educativa en las personas mayores, viendo como ésta afecta tanto a la calidad de vida como a la salud o el ocio de estos ciudadanos. Finalmente, en los años 80 con el desarrollo de los Centros de Educación de Personas Adultas (EPA), las Aulas de la Tercera Edad y los Programas Universitarios para Mayores (PUM) se consolida en Europa la tercera generación de programas socioeducativos para personas mayores, donde se am- 
Principios educativos ocio humanista en los... | M..$\underline{a}$ Luisa Amigo Fernández de Arroyabe et al.

plían temas y programaciones, generan y difunden conocimientos, participación en aprendizajes autodirigidos y en proyectos de investigación.

Las iniciativas pioneras en España para responder desde las instituciones universitarias a las demandas de una educación para el colectivo de mayores, son, además del Instituto de Estudios de Ocio que tomaremos como referencia, las Universidades de Alcalá de Henares, la Universidad Pontificia de Salamanca y la Universidad de Granada. Consolidadas estas experiencias, poco a poco y de forma constante, siguen surgiendo distintos proyectos universitarios a nivel estatal, llevando todo ello a la creación, en febrero de 2004, de la "Asociación Estatal de Programas Universitarios para Mayores (AEPUM)», con el fin, tal como recoge en sus Estatutos, de fomentar los programas educativos para mayores en el ámbito universitario, contribuyendo al desarrollo formativo y cultural de dicho colectivo. La Asociación tiene como objeto el fomento y desarrollo del diálogo y comunicación entre las Universidades, las Administraciones Públicas y entidades privadas, impulsando la cultura y la búsqueda de soluciones para la satisfacción de necesidades formativas. Se propone actuar como centro de información y asesoramiento interuniversitario para los Programas de Mayores, adecuándose en su actuación a los principios de autonomía, participación y relación con el entorno.

La acción de la Asociación, según datos del curso 2014-15, afecta a más de 43.000 alumnos mayores aproximadamente, repartiéndose de la siguiente manera: un $65 \%$ de mujeres y un 35\% de hombres. En este momento la integran 43 universidades repartidas por las comunidades autónomas. Hay que apuntar que existen también programas en otras Universidades del Estado pero que actualmente no pertenecen a la Asociación. Entre los fines que se plantea la Asociación está el concertar con las Administraciones Públicas estudios y proyectos que sean de interés para la formación permanente en el ámbito de los programas para mayores.

Aunque es en 2004 cuando nace la Asociación, desde 1996 se vienen produciendo encuentros nacionales en relación a estos programas, habiéndose abordado a lo largo de los años temas de interés para el desarrollo de los mismos. Igualmente desde 1998 se han ido realizando Jornadas Científicas y Técnicas, así como Seminarios que han permitido ir avanzado en aspectos clave en el desarrollo de los PUM. Otro reto importante de esta Asociación de Programas Universitarios para Mayores ha sido la investigación. En sus objetivos se recoge que se fomentará la investigación y publicación de trabajos científicos, estudios, monografías y tesis doctorales en el ámbito de la formación de las personas mayores, puesto que la generación de conocimiento es imprescindible para el desarrollo de los mismos, en particular y de la sociedad, en general.

Durante la primera década del siglo xxi se trabajó fundamentalmente en los «ModelosMarco de PUM's», orientando parte de su trabajo y esfuerzo a la elaboración de una propuesta de los mismos. Para ello se elaboró una encuesta de referencia remitida a los Programas y Universidades de los que constaban datos oficiales y fidedignos, que consti- 
Principios educativos ocio humanista en los... | M..$\underline{a}$ Luisa Amigo Fernández de Arroyabe et al.

tuyeron la base de una investigación posterior recogida en la publicación Bru (2002). También en 2006, desde la Universitat Jaume I (Cabedo y Alfageme, 2006) se desarrolló una investigación sociológica de los PUM en España, abordándose los objetivos, la organización, contenidos y otros elementos que muestran la unidad y diversidad de los PUM, así como todo lo relativo al profesorado. También se exponen las características sociodemográficas del alumnado así como sus actitudes y opiniones en relación al desarrollo de los programas. Por último, y no de menor importancia, otra línea de investigación que viene ocupando a las distintas universidades son los diferentes procesos de enseñanza-aprendizaje que se desarrollan en los PUM, así como la evaluación de los mismos.

El Instituto de Estudios de Ocio de la Universidad de Deusto, que tomamos como referencia de análisis conceptual de los programas señalados hasta aquí, se desarrolla paralelamente a esta corriente de las universidades públicas, a partir de la investigación y la experiencia centrada en programas para adultos, sin el etiquetado de Programa de Mayores. Sin embargo, la nota clave que caracteriza a estos Estudios procede de su concepción inicial de la formación como ocio, implícita en la línea de investigación «el ocio como desarrollo humano» y que dará como resultado la teoría de ocio humanista, que constituye la base del análisis que sigue.

\section{FUNDAMENTACIÓN TEÓRICA. EL MODELO DE OCIO HUNANISTA}

En este apartado nos proponemos analizar los fundamentos teóricos que sustentan el modelo de formación como ocio. Lo haremos de forma sintética ordenando los principios y valores que lo vertebran, destacando las notas clave de la comprensión del ocio humanista. La fundamentación bibliográfica de este modelo se concreta en diversos estudios, especialmente en los cerca de cincuenta volúmenes de Documentos de Estudios de Ocio, publicados en la Universidad de Deusto, así como en diferentes tesis, tesinas, trabajos fin de máster y otros proyectos de investigación que se han realizado en el Programa de Doctorado Ocio y Desarrollo humano y en la actualidad, Ocio, cultura y comunicación para el desarrollo humano (http://www.ocio.deusto.es/publicaciones).

\subsection{Ocio humanista}

Centramos la atención no en el ocio como fenómeno general sino en el ocio humanista: «Una experiencia humana (personal y social) integral, motivada por la vivencia intencional del ocio autotélico, entendido como Derecho humano y ámbito de desarrollo, al que se accede mediante la formación» (Cuenca, 2010: 66). Se caracteriza por su multidimensionalidad y por sus actuaciones queridas, satisfactorias y con un fin en sí mismas, lo que subraya el autotelismo. Este ocio favorece el desarrollo personal y social (Kleiber, 2000, 2012; Kleiber et al., 2011) siendo un ámbito de valor, un ocio positivo que se integra en modos de vida y potencia valores personales y comunitarios (Nimrod y Adoni, 2006; Stebbins, 2006; 
Principios educativos ocio humanista en los... | M..$\underline{a}$ Luisa Amigo Fernández de Arroyabe et al.

Silvestre, 2000; Cuenca, J. 2012). Debemos considerarlo integrado en la vida de las personas (Cuenca, 2000; Cuenca, 2014). El ocio humanista se reconoce como un ocio positivo y digno desde la antigüedad (Séneca, 1922; Cicerón, 1994), que favorece la mejora de la persona y la comunidad. Se sustenta en tres valores fundamentales de todo ocio: libertad, satisfacción y gratuidad o autotelismo, orientándose hacia referentes de identidad, superación y justicia. Se puede entender como un ocio personal o un ocio comunitario, pero, en cualquier caso, no es un ocio espontáneo, sino una experiencia integral compleja que requiere formación.

En este trabajo nos interesa destacar su potencialidad para la formación, de tal modo que la experiencia de ocio puede ser más valiosa gracias a la capacitación (Faché, 2002; Hutchinson y Robertson, 2012). La formación permite el desarrollo de valores, actitudes y destrezas de ocio, mejorando la calidad de vida de las personas.

En definitiva, el ocio humanista es un ocio valioso, experiencial, abierto, activo, creativo, sustancial, solidario, que da sentido, ayuda a mejorar y permite la realización de valores. Este rasgo incide claramente en el tema que estudiamos en estas páginas sobre los programas de formación de adultos. Nos detenemos, a continuación, en las categorías esenciales de la comprensión del ocio humanista: experiencia, libertad, satisfacción y autotelismo.

\section{Experiencia}

La comprensión del ocio humanista como un área específica de la experiencia humana, ámbito de desarrollo personal y social, ha favorecido la primacía de la vivencia, privilegiada en su carácter personal, emocional y procesual. Personal en cuanto que el ocio hunde sus raíces en la dimensión humana del ser que proyecta, que tiene que realizar su vida y que debe elegir. Esta misión la lleva a cabo en una relación con el entorno y requiere una apertura comprometida de la persona. La vida humana es proyecto, creación, invención y el ocio forma parte de este proyecto. El ocio como experiencia se deslinda de su comprensión como actividad y no se confunde con ella (Cuenca, 2004: 195 y ss.). Despliega su riqueza en el proceso de realización temporal en el que pasado, presente y futuro integran un continuo dilatado de experiencia y conciencia. Esta resonancia en la subjetividad abre la vivencia al sentido y posibilita el despliegue de comprensión de la misma, superando así la inmediatez de una emoción psicofísica.

En síntesis: la experiencia de ocio es una vivencia humana subjetiva, libre, satisfactoria y con un fin en sí misma, que se caracteriza por su carácter procesual, estar integrada en valores, tener un predominio emocional, no justificarse por el deber y estar condicionada por el entorno en el que se vive.

\section{Libertad}

El ocio humanista arraiga en la libertad, entendida como libertad de y libertad para (Cuenca, 2004: 252). Si la primera indica ausencia de coacción, independencia de elección, 
Principios educativos ocio humanista en los... | M..$\underline{a}$ Luisa Amigo Fernández de Arroyabe et al.

la segunda tiene el sentido positivo de la capacidad de elegir en cada momento el modo de obrar que se considere mejor entre las distintas posibilidades que se ofrecen. Frente a un ámbito de necesidad, el ocio se comprende como un horizonte de libertad que se opone al automatismo y la pasividad, y requiere una voluntad de participar, de construir activamente. En el ámbito de la experiencia de ocio se destaca la voluntariedad del sujeto sobre la base de ciertas condiciones culturalmente condicionadas, «de ciertas pautas sociales que a la vez limitan y hacen posible su interpretación» (Cuenca, 2012: 230). Esta libertad se desarrolla con la educación y es un pilar clave en los programas de formación, no pensando en favorecer la elección entre diversos productos ofertados, sino, sobre todo, la elección de caminos y procesos, en facilitar itinerarios de ocio (Monteagudo y Cuenca, 2012). Ellos son los que posibilitan a la larga el desplegar la libertad que permite disfrutar.

\section{Satisfacción}

La satisfacción o el disfrute se comprende como una nota clave de la experiencia del ocio humanista y, por lo tanto, un pilar fundamental de la educación del ocio. No está reñida con el esfuerzo ni debe confundirse con lo meramente placentero (Cuenca, 2004: 254). Arraiga en la subjetividad de la persona y se concreta en un ajuste entre sus expectativas y la vivencia de ocio real (Csikszentmihalyi, 1998). Este principio ha impulsado la acción educativa compatibilizando el disfrute, la creación y el aprendizaje. Favorece que la persona experimente goce en la realización de la práctica formativa y que aprenda a disfrutar aumentando su potencialidad creativa. Se ha comprendido también como un antídoto contra los ocios nocivos que, lejos de satisfacer necesidades personales, generan dependencia e imposibilitan el desarrollo de la persona.

\section{Autotelismo}

El ocio es una experiencia humana libre, satisfactoria y con un fin en sí misma; es decir, voluntaria y separada de la necesidad, entendida como necesidad primaria. Estamos destacando que la experiencia de ocio se caracteriza por ser una percepción subjetiva en la que influyen tanto la propia experiencia personal como el entorno social en el que se vive (Cuenca, 2010: 67). El ocio humanista es un ámbito de valor en sí mismo, sin la búsqueda de la utilidad y, por tanto, se caracteriza por la gratuidad. Esta concepción favorece que las personas sean capaces de apreciar y cultivar el ocio por su valor autónomo, junto con el deseo de superación y reto, propio de la acción libremente elegida. Se trata de un ocio gratuito, en cuanto que no busca la utilidad, aunque proporciona autorrealización y calidad de vida. Es referente de todo ocio positivo, de ahí que se considere el punto de partida del ocio humanista.

Estos principios esbozados llevan consigo otros que provienen de la educación y que se consolidan como notas clave de la formación como ocio. Nos referimos a la persona como centro de desarrollo en el marco de un proceso a lo largo de la vida. 
Principios educativos ocio humanista en los... | M..$\underline{a}$ Luisa Amigo Fernández de Arroyabe et al.

\section{La persona como eje de desarrollo en el marco de un proceso a lo largo de la vida}

El punto central del ocio humanista está en la persona y en su desarrollo como proceso a largo plazo. Potencia valores personales y comunitarios. El principio de personalización hace compatibles el ocio individual y el ocio social compartido, abriendo el horizonte al ocio solidario, a la responsabilidad y el compromiso. La educación personal del ocio defiende el desarrollo de una persona libre e independiente, pero también integrada y solidaria con las demás. Su gran reto, a la hora de llevar a la práctica este principio, es orientarlo hacia experiencias valiosas de formación, tratando de que las personas sean capaces de obtener las máximas posibilidades y beneficios de estas experiencias (Cuenca, 2010: 123). En este sentido destacan también los valores de identidad y autorrealización, así como de superación (Cuenca, 2011: 38-41).

Este principio se orienta a la realización del aprendizaje a lo largo de la vida (Kleiber, 2002), resaltando el ocio como un eje vertebrador de desarrollo personal. Supone la base de lo que podemos considerar un ocio serio (Stebbins, 1992). La finalidad sería el logro de experiencias de ocio valiosas en sí mismas, que generen la continuidad. Se encamina a fortalecer interiormente a las personas participantes, facilitándoles la interiorización de conocimientos, valores, actitudes y destrezas básicas de ocio que les permitan realizarlo de la manera más óptima posible. Se pretende alentar la capacidad de las personas para encontrar o crear por sí mismas los medios para aprovechar sus posibilidades de ocio, tratando de implicarlas en un esfuerzo personal significativo.

La educación del ocio comprendida como un proceso continuo de aprendizaje a lo largo de la vida supone una acción educativa no casual, sino proyectada en el tiempo, con unos objetivos y estrategias adaptados a las necesidades y a las posibilidades de actuación (Cuenca, 2004: 256). Se destaca así la coherencia y la proyección de la acción educativa en el continuo vital de la persona. Supone un proceso de mejora que lleva consigo el que cada persona interiorice la formación para que, a su vez, pueda abordar con éxito nuevas experiencias (Caride, 2012). La formación dista de ser utilitaria y pragmática, como en otros tramos, y propicia que la persona se implique sin la necesidad de recompensas, logros tangibles o reconocimiento social; por tanto, puede vivirse como una experiencia de ocio en su dimensión formativa (Gómez y Martínez, 2002).

La finalidad es favorecer el desarrollo integral de la persona a partir de conocimientos, actitudes, habilidades y valores de ocio. La consecuencia esperada es capacitar a las personas para aumentar la calidad de vida mediante el ocio y la autoorganización. Se desea potenciar al máximo las posibilidades creadoras, físicas e intelectuales como desarrollo personal y para el enriquecimiento de la comunidad (Cuenca, 2014).

El ocio experiencial destaca el papel fundamental de la persona en la elección y en la perseverancia. Aunque el ocio puede ser una vivencia espontánea sin más, la experiencia de 
Principios educativos ocio humanista en los... | M..$\underline{a}$ Luisa Amigo Fernández de Arroyabe et al.

ocio humanista requiere capacitación. Esta capacitación resulta más evidente cuando se trata de las experiencias de ocio cultural (Cuenca, 2010). Los programas deberían centrar sus propuestas metodológicas educativas en la vivencia intencional del proceso experiencial del ocio. Incitar, estimular, ayudar, familiarizar con las posibilidades de ocio deberían ser acciones presentes en las propuestas didácticas, tratando de favorecer el desarrollo de las personas participantes.

\section{Un ocio digno}

El ocio es un valor en sí mismo y un Derecho que conlleva equidad y dignidad, por tanto, solidaridad, inclusión y sostenibilidad. Desde el punto de vista comunitario, afirma los valores básicos de la ciudadanía y la convivencia. El ocio digno se realiza a través del reconocimiento del derecho al ocio, que desde el punto de vista comunitario, lleva consigo la supresión de barreras, sostenibilidad e inclusión (Cuenca, 2011).

El principio de inclusión asume que la convivencia y el aprendizaje en los grupos con diferencias son la mejor forma de beneficiar a todos y a cada uno (Cuenca, 2004: 258). La formación como ocio es una nueva oportunidad de desarrollo e integración de las personas y debe favorecer la sociabilidad a través de la participación. Los programas del Instituto se han caracterizado por facilitar esta integración con el resto de estudiantes universitarios, compartiendo espacios y servicios. Destacamos también la convivencia multigeneracional y el respeto a la heterogeneidad. Se ha adoptado como principio la equiparación de oportunidades y se ha potenciado una comunidad incluyente, que reconoce diferencias y favorece la inclusión. La aceptación de la diferencia es un valor para que se dé la plena inclusión de todos los alumnos (Lázaro, 2004). La dimensión solidaria se concreta en responsabilidad, respeto y sostenibilidad (Cuenca, 2005). En definitiva, un ocio digno sólo se puede llevar a cabo a través de un ocio justo e inclusivo.

\subsection{Los programas de formación permanente del Instituto de Ocio: un apunte}

Los programas del Instituto de Estudios de Ocio de la Universidad de Deusto se fundamentan en el concepto de ocio humanista, cuyos principios acabamos de esbozar. Esta teoría ha generado muchos estudios en los veintiocho años de vida del centro y ha promovido la realización de este concepto en el ámbito de la investigación, de la formación y, especialmente, en la oferta de educación comunitaria. Entre las investigaciones realizadas destaca «Claves para la intervención ciudadana en Ocio, con un enfoque educativo, en Vitoria-Gasteiz» (2011-12), el Proyecto ITINERE (Plan Nacional I+D+I, 2012) del que forma parte este estudio y que ya ha sido citado, así como los desarrollados los últimos años para la Diputación Foral de Bizkaia en torno a «Propuestas de optimización de la intervención pública para un envejecimiento activo a través del ocio en Bizkaia»(2014); «Ocio y envejecimiento activo satisfactorio» (2013), «Intervenciones de promoción del envejecimiento activo como estrategia de prevención de la discapacidad y dependencia en personas mayores en Biz- 
Principios educativos ocio humanista en los... | M..$\underline{a}$ Luisa Amigo Fernández de Arroyabe et al.

kaia» (2012), entre otros. Se ha considerado deseable y con esta finalidad se han implementado diversos programas desde 1993, para que los participantes puedan ampliar el horizonte cultural, descubrir valores y buscar en el ocio nuevos cauces de realización personal (Cuenca, M., Lázaro, Amigo, Cuenca, J., 2012).

Desde el primer momento, con la puesta en marcha del programa Ocio cultural universitario en 1993, se tuvo la intención de ofrecer la formación como ocio, tratando de que esta oferta fuera un ámbito de posibilidad de desarrollo personal y social (Cuenca, 2004). Con el transcurso del tiempo, los programas que se implementan en 2008-2009, Disfrutar las Artes y Aprender Viajando, aspiran a ser ellos mismos un espacio de realización de experiencias de ocio, acentuando la dimensión vivencial y promoviendo la dimensión creativa, especialmente en su faceta recreativa. Por otra parte el titulado en Cultura y solidaridad se implementa en el curso 1999-2000. Todos estos programas nos ayudan a valorar los pilares que se han considerado claves y acordes con la orientación humanística del Instituto. Uno de ellos es el punto central de la formación como ocio.

Desde el inicio se orientó la formación permanente de adultos desde esta idea directriz que conjugaba la formación integral de la persona y su desarrollo a partir de experiencias culturales de ocio, con una metodología de enseñanza que hiciera posible la formación como ocio (Cuenca, 2009). Se puso especial atención en la elección del profesorado, elegido por su pasión por el tema, así como por su entusiasmo comunicador; ambos aspectos favorecían la realización del modelo vivencial. Este proyecto se incrementa conscientemente en los programas Disfrutar las artes y Aprender viajando, en los que el carácter experiencial está en el mismo núcleo de su orientación. En ambos se intenta favorecer una experiencia enriquecida con el conocimiento, tanto en el encuentro con las artes como con los lugares vivos de la cultura. Disfrutar las artes se ha orientado al gozo y el disfrute de las artes, vinculado a su conocimiento y a su comprensión. Se ha optado también por un encuentro con los artistas, como espacio privilegiado de experiencia de ocio estético y de comunicación. La formación como experiencia de ocio se manifiesta en el doble nivel que hemos comentado, en el aula y diacrónicamente a lo largo del ciclo. Muchas clases son una realización de estas experiencias y los programas la favorecen en la medida de lo posible. Pero también lo es a largo plazo y, en este sentido, el desarrollo de los tres años de Cultura y solidaridad supone la base de lo que podemos considerar un ocio serio (Stebbins, 1992, 2008).

Los programas favorecen la libertad promoviendo en el estudiante la elección de materias desde una oferta amplia y variada. Se inscriben en los programas como una elección libre y desinteresada, pues no buscan una formación profesional. Con frecuencia se les anima a que participen en las clases con textos personalmente elegidos, fomentando la preparación de la experiencia. Se les anima a buscar en sus gustos y a comunicarlo a los demás, a buscar nuevos ámbitos de disfrute y a explorar nuevos itinerarios de ocio. La elección personal, la apertura de miras, el planteamiento siempre novedoso de las artes son aspectos que el profesorado tiene siempre en cuenta. 
Principios educativos ocio humanista en los... | M..$\underline{a}$ Luisa Amigo Fernández de Arroyabe et al.

Quizá sea la satisfacción una de las notas que destaca más en el horizonte de los participantes. Estos estudiantes anhelan una experiencia, llevada a cabo con disfrute y satisfacción, junto con otros alumnos con los que no compiten, sino que comparten expectativas. Disfrute y satisfacción son términos reiterados por ellos y por los profesores. Los participantes de Cultura y solidaridad, especialmente, confirman la autosuperación individual gozosa, en el marco de un tiempo de tres años que comparten con un grupo. La orientación general de los programas hacia el disfrute ha sido una constante desde el inicio. Se ha tenido en cuenta que se puede aprender a disfrutar acercando a los estudiantes a campos que anteriormente les eran desconocidos o ajenos, aumentando su potencialidad creativa. El conocimiento es aquí un punto central que intensifica el goce y lo favorece. Disfrutar las artes nació, especialmente, con esta intención y así se mantiene, deseando hacer realidad esa experiencia satisfactoria, privilegiando favorecer el disfrute de las artes mediante la educación estética y el conocimiento (Amigo, 2014).

Los programas ofrecen la formación destacando su valor, deslindado de la utilidad. La nota clave aquí es el autotelismo. Se elige libremente sin buscar otra meta, destacando el sentido de la propia experiencia, enmarcada en el seno de la tradición humanística. El logro se consigue como un fin en sí mismo, no como un medio para la preparación laboral, sino con una orientación autotélica que da valor al cultivo de la persona. En el marco de este horizonte se hace ver a los participantes que la cultura está abierta al sentido, a pensar y tratar de comprender el mundo humano. Las experiencias autotélicas, que tienen en sí mismas el beneficio de su propia realización son, al mismo tiempo, un ámbito de apertura a los otros. Algunos estudiantes destacan la oportunidad de poder ir a la universidad, con lo que ello significa en su ampliación de su visión del mundo y del entramado de relaciones sociales. En este sentido los programas del Instituto favorecen este encuentro con otras personas con las que los estudiantes pueden compartir inquietudes y anhelos.

El planteamiento de los programas se orienta a la realización de un ocio experiencial valioso a largo plazo. Todos ellos facilitan competencias y actitudes para que los estudiantes puedan disfrutar de productos culturales complejos. Esta apuesta lleva consigo que el ocio se integre en sus valores y modos de vida. Se favorece la integración del ocio en el ámbito personal y se busca abrir la mirada haciendo a los participantes más sensibles a los problemas sociales. Un punto central en el recorrido del Instituto es la atención a la discapacidad y, desde luego, a la heterogeneidad que se manifiesta a diario en las aulas. Hemos destacado algunos valores que se favorecen en el desarrollo de los programas tales como la integración, la convivencia multigeneracional, el respeto a la heterogeneidad, la solidaridad y el fomento del disfrute del hecho cultural. 


\section{LECTURA DE LOS PROGRAMAS UNIVERSITARIOS PARA MAYORES DE LAS UNIVERSIDADES ESPAÑOLAS DESDE EL HORIZONTE DE LA TEORÍA DEL OCIO HUMANISTA}

Nos proponemos, a continuación, presentar la lectura de los Programas Universitarios para Mayores, desde el punto de vista de su implementación como proyectos de ocio humanista, antes reseñado. Antes de hacerlo, conviene especificar la metodología que se ha seguido para realizar este estudio.

\subsection{Metodología}

Siendo conscientes de las limitaciones de este trabajo, se ha optado por una metodología sencilla pero clara que consiste en confrontar las categorías fundamentales de la teoría del ocio que se han fundamentado en las páginas precedentes con un análisis de contenido de los documentos sintetizados públicamente en la información mostrada en la página web de cada centro.

Se han consultado, concretamente, las páginas de 35 universidades españolas (ver anexo) para realizar una interpretación hermenéutica de sus planteamientos de los Programas Universitarios para Mayores, tal como allí aparecen, prestando especial atención a tres elementos: sus justificaciones generales, sus objetivos y el contenido concreto de los programas. Para leerlos desde la teoría del ocio, se han rastreado en ellas las referencias a las seis siguientes categorías, que responden a los principios de la teoría y corresponden a la fundamentación teórica del ocio humanista:

Tabla 1. Categorías que configuran la Teoría del Ocio Humanista

\begin{tabular}{|c|c|c|c|c|c|}
\hline Ocio humanista & Experiencia & Libertad & Satisfacción & Autotelismo & $\begin{array}{c}\text { Personalización y } \\
\text { valores }\end{array}$ \\
\hline $\begin{array}{l}\text { Promover el ocio } \\
\text { humanista. } \\
\text { Promover ocio } \\
\text { valioso experien- } \\
\text { cial }\end{array}$ & $\begin{array}{l}\text { Promover ex- } \\
\text { periencias de } \\
\text { aprendizaje: } \\
\text { - Diversas. } \\
\text { - Globales (todo } \\
\text { se relaciona } \\
\text { con todo). } \\
\text { - In tegradas } \\
\text { (relacionadas } \\
\text { con el sentido } \\
\text { de la vida y } \\
\text { los valores de } \\
\text { cada uno). }\end{array}$ & $\begin{array}{l}\text { Promover ex- } \\
\text { periencias de } \\
\text { aprendizaje li- } \\
\text { bre. } \\
\text { - Sin coacción. } \\
\text { - Sin direccio- } \\
\text { nismo ni pa- } \\
\text { ternalismo. } \\
\text { - Que estimulen } \\
\text { y abran ca- } \\
\text { minos de de- } \\
\text { sarrollo perso- } \\
\text { nal. }\end{array}$ & $\begin{array}{l}\text { Promover ex- } \\
\text { periencias de } \\
\text { aprendizaje: } \\
\text { - Gratifican- } \\
\text { tes tanto en } \\
\text { el proceso de } \\
\text { aprendizaje } \\
\text { como en sus } \\
\text { resultados. } \\
\text { Que tiendan a } \\
\text { ajustar las ex- } \\
\text { pectativas de } \\
\text { los participan- } \\
\text { tes y la reali- } \\
\text { dad. }\end{array}$ & $\begin{array}{l}\text { Promover ex- } \\
\text { periencias de } \\
\text { aprendizaje: } \\
\text { - Valiosas en sí } \\
\text { misma. } \\
\text { - Que supongan } \\
\text { un reto de au- } \\
\text { tosuperación } \\
\text { respecto expe- } \\
\text { riencias ante- } \\
\text { riores. }\end{array}$ & $\begin{array}{l}\text { Promover ex- } \\
\text { periencias de } \\
\text { aprendizaje: } \\
\text { - Que partan de } \\
\text { los sujetos. } \\
\text { - Que tengan } \\
\text { objetivos in- } \\
\text { dividuales y } \\
\text { grupales. } \\
\text { - Que favorez- } \\
\text { can la perso- } \\
\text { nalización. } \\
\text { - Que se vincula } \\
\text { a valores y al } \\
\text { sentido. }\end{array}$ \\
\hline
\end{tabular}

Fuente: Elaboración propia a partir de Cuenca (2004: 251-260) y Amigo-Cuenca (2012: 431-432). 
Principios educativos ocio humanista en los... | M..$\underline{a}$ Luisa Amigo Fernández de Arroyabe et al.

Como prevención metodológica y para hacer posible el análisis tal como se ha planteado, ha sido preciso solventar algunas diferencias en el vocabulario empleado. Así, por ejemplo, en el caso del concepto autotelismo. Se trata de un término poco utilizado y, sin embargo, entendido como promoción de experiencias de aprendizaje valiosas en sí mismas (Cuenca, 2004: 2010). Es decir que, con un criterio amplio, se puede reconocer prácticamente en todos los programas. Por el contrario, se ha respetado literalmente la presencia o ausencia del principio de satisfacción, registrando los programas que textualmente incorporan el disfrute o el gozo como elemento clave en el proceso de aprendizaje. No es el mismo caso que con el término autotelismo; mientras que el uso de éste es más restringido, la acepción de satisfacción es de uso común. El término experiencia, entendido como promoción experiencias de aprendizaje diversas, globales, integradas, relacionadas con el sentido de la vida y los valores de cada uno, en suma, como experiencias de desarrollo personal y social (Cuenca, 2004: 2010), se recoge prácticamente en todos los programas universitarios.

Como resultado del estudio, se mostrará una sencilla ficha de presentación de resultados, en la que puede observarse la lectura realizada esquematizada con una X. Somos conscientes de la limitación del análisis que podría enriquecerse con otras metodologías, como entrevistas en profundidad a los responsables, que complementarían lo que aquí se apunta. Sin embargo, dada la restricción espacio-temporal de este trabajo, presentamos este estudio de la implementación de las categorías como un modo de abrir camino localizando las ideas generales y con el deseo de que se amplíe con nuevas líneas de investigación futuras, algunas ya iniciadas.

\subsection{Análisis de los programas}

Como se apuntaba más arriba, haciendo una lectura flexible de algunas de las categorías estudiadas se certifica su presencia en los planteamientos de la mayor parte de los programas. Así, por ejemplo, la práctica totalidad de ellos buscan ofrecer una experiencia valiosa a sus alumnos, incidiendo especialmente en la idea de promover el desarrollo personal en relación con la formación. Profundizando más en este concepto hallamos que se despliega en algunas direcciones significativas. Como cabía suponer, pues forma parte de la esencia de este tipo de acción educativa, una de las más frecuentes es la idea de aprendizaje a lo largo de la vida o formación continua, que se presenta de manera explícita en diez de los casos, aunque se puede comprender como justificación global de prácticamente todos ellos.

Otros conceptos interesantes relacionados con el ámbito experiencial son los de intercambio e intergeneracionalidad. Si bien aparecen en menos casos, ofrecen una perspectiva valiosa, ya que enriquecen el concepto de experiencia que se ofrece a los participantes. Así, algunas universidades citan la idea de intercambio «de conocimientos y experiencias de tipo cultural» (Universidad de Vigo, en fórmula muy similar a la Universidad Jaume I), en el sentido de que no sólo los alumnos se benefician de lo que los programas les ofrecen, 
Principios educativos ocio humanista en los... | M..$\underline{a}$ Luisa Amigo Fernández de Arroyabe et al.

sino que se da también una «retroalimentación del sistema educativo a través de personas ya formadas y expertas» (Universidad de Murcia). En relación estrecha con esto, ocho universidades explican directamente que sus programas quieren ser intergeneracionales; de este modo el concepto de experiencia se amplia, pues no sólo define la que los participantes obtienen de la universidad, sino la que ellos ofrecen a ésta.

En relación al concepto de libertad, como se observa en la tabla, es también muy frecuente y solamente en ocho programas no aparece citado abiertamente entre sus objetivos. Ahora bien; en la gran mayoría de los casos, al menos veinte según los datos recogidos, este concepto se concreta en la optatividad, es decir, en la posibilidad que se ofrece al alumno para diseñar su propio itinerario en el programa. Hay diferentes grados de optatividad que se pueden referir a parte de las asignaturas, incluso a todas ellas, como en el caso de la Universidad de Murcia, o al itinerario en su conjunto. Algunos programas (Universidad Rovira i Virgili, Universidad Autónoma de Barcelona, Universidad de Málaga) permiten a sus alumnos escoger entre ser o no evaluados por los profesores según su rendimiento. De acuerdo a nuestra definición, entonces, se cumple muy significativamente con el objetivo de ofrecer una experiencia de aprendizaje sin coacción, sin determinismo ni paternalismo. Además, en algún caso encontramos expresiones que se hacen conscientes de que esto puede abrir caminos de desarrollo personal; por ejemplo, la Universidad José Saramago propone la "participación activa del alumno en el proceso de aprendizaje» y la de Murcia quiere facilitar «el conocimiento, los procedimientos y la metodología necesarios para el correcto desarrollo de un aprendizaje autónomo».

Es, sin embargo, la categoría de satisfacción la que con menor frecuencia se refleja en los planteamientos revisados. Únicamente en cuatro de los programas hemos hallado referencias a ella. La Universidad José Saramago se refiere las personas que «encuentren placer en el estudio y el conocimiento" y les presenta una alternativa para experimentarlo; la Universidad de Comillas ofrece «disfrutar del aprendizaje»; la Universidad de CEU insiste en ello añadiendo «durante toda la vida», en el ámbito de la formación continua; finalmente, la Universidad de Oviedo, de modo más proactivo, pretende «alentar el gusto por el conocimiento y la cultura».

Es bien visible que la idea de satisfacción que manejamos desde la teoría del ocio humanista no se está teniendo en cuenta al elaborar los PUMs en las universidades españolas. Sin embargo, sí hemos localizado algunas alusiones a conceptos cercanos aunque de diferente matiz como bienestar o calidad de vida, que, si se incluyen en el campo de la satisfacción, podrían dar la impresión, no coherente con los propósitos reales de los programas, de derivar hacia lo asistencial más que hacia lo formativo. Por ello, hemos preferido tomar tales conceptos, que aparecen en ocho ocasiones, como muestras de la lectura amplia del autotelismo antes descrita. Nos anima a ello el comprobar que aquí aparecen vinculados con la idea de la promoción personal a través del conocimiento, como ocurre significativamente en el caso de la Universidad de las Islas Baleares, que persigue promocionar «la calidad de vida de las personas mayores a través del conocimiento y de las relaciones que 
Principios educativos ocio humanista en los... | M..$\underline{a}$ Luisa Amigo Fernández de Arroyabe et al.

se establecen en el ámbito universitario». Así, bienestar o calidad de vida forman parte de un concepto flexible de autotelismo, en ningún caso citado explícitamente en este sentido técnico en el que nos lo presenta la teoría del ocio humanista, pero reconocible en expresiones que definen la promoción de experiencias de aprendizaje valiosas en sí mismas. Esta idea la encontramos conectada, por lo tanto, con el ámbito experiencial y con el del conocimiento. Se manifiesta, en el primer caso, en propósitos como fomentar la autosuperación (Universidad de Santiago, Universidad Pontificia de Salamanca), la autoafirmación personal (Universidad de Comillas), el crecimiento personal (Universidad de Salamanca, Universidad Ramon Llull) y otras expresiones de sentido similar. Por otro lado, en cuanto al valor del conocimiento, aparecen objetivos que resaltan la «extensión educativa y cultural en un contexto social y universitario» (Universidad de Santiago), el «aprendizaje como cauce privilegiado del conocimiento y el compromiso» (Universidad de Comillas) o la «emancipación social y cultural de las personas» (Universidad Jaume I).

En todo caso, resulta claro que el concepto más preciso de actividad autotélica, ajustado a la investigación desde el ocio humanista, puede aplicarse sólo limitadamente a los programas estudiados, mientras que, en la forma más amplia que hemos tomado como referencia, se aplica a la gran mayoría de los casos (sólo lo hemos encontrado ausente en nueve de ellos, incluidos aquéllos en los que la información disponible era muy escasa). Lo que permite suponer que una aplicación más exacta permitiría aclarar propósitos que desde luego están latentes en los planteamientos de las universidades, pero que se manifiestan a través de conceptos que se escoran hacia el campo de la experiencia o de los valores.

Esto se confirma porque precisamente en este ámbito que identificamos como personalización y valores encontramos un fuerte énfasis: sólo seis de los programas no ofrecen alguna reflexión al respecto y los conceptos presentes son muy variados, si bien giran en torno a dos ideas básicas: el desarrollo y enriquecimiento personal y la relación con la sociedad. Más concretamente, localizamos algunas líneas clave: en primer lugar, la que se refiere a la integración, palabra que vemos reflejada hasta en diez ocasiones, aunque está implícita en otras más. Tal idea se despliega en varias direcciones: integración de los mayores a la universidad con la consiguiente integración social e incluso en un caso europea. El mismo propósito general de los programas se define, ya en el funcionamiento interno de los mismos, como un objetivo muy repetido (hasta en once ocasiones) de interacción, ya sea como fomento del trabajo en grupo, como relación de los alumnos entre sí, con los profesores y la comunidad universitaria o como encuentro intra e intergeneracional, un valor que ya pusimos en relación con el campo de la experiencia y que tiene cabida también en este punto. En una segunda orientación, se reitera en ocho ocasiones de manera explícita la necesidad de contribuir a la adaptación de los participantes a los cambios sociales y a las nuevas tecnologías. Finalmente, es muy frecuente, aunque se exprese de modos variados, la referencia a la sociedad como objetivo: en siete de los programas se pone como meta la participación o participación social de los alumnos a través de su proceso formativo; tres de ellos se proponen contribuir a la ciudadanía activa y la solidaridad; dos hablan, de modo más abstracto, de favorecer las relaciones sociales y en seis ocasiones se de- 
Principios educativos ocio humanista en los... | M..$\underline{a}$ Luisa Amigo Fernández de Arroyabe et al.

fiende que la universidad cumple, a través de los PUMs su compromiso con la sociedad o que devuelve con ellos a los mayores su aportación a la sociedad. La Universidad de Extremadura, por ejemplo, se propone «corresponder a la aportación a la sociedad en forma de bienes culturales».

Además de la localización de las categorías fundamentales que identificamos desde la teoría del ocio humanista, hemos tenido ocasión de encontrar algunas referencias explícitas al propio concepto de ocio. Si bien son escasas, merece la pena señalarlas, ya que inciden precisamente en nuestra línea de estudio. Así, la Universidad de Extremadura tiene entre sus objetivos el «fomento del empleo creativo del ocio", fórmula casi idéntica a la de la «Universidad de Huelva», mientas que la Universidad de Granada reconoce que «los alumnos tienen el deseo de convertir el ocio en una oportunidad transformadora» y la Universidad de Murcia «promover un mejor conocimiento del entorno para el aprovechamiento de las ofertas de ocio (culturales, físicas, intelectuales) que ofrece la sociedad». La Universidad de Oviedo plantea la fórmula más precisa: «Formar para el ocio activo y recreativo». Todo ello se aproxima a la interpretación que aquí hemos propuesto de acuerdo a los avances en el estudio del ocio humanista.

En conjunto, además de estas últimas interesantes referencias directas, la presencia frecuente en la información de los diferentes programas de objetivos, principios y valores que hemos identificado de modo más o menos flexible con las claves del ocio humanista nos llevan a concluir, primero, que tales principios están de hecho en la base de las propuestas universitarias para los PUMs y, segundo, que, en consecuencia, una aplicación más explícita de la teoría permitiría clarificar eficazmente los planteamientos haciendo visibles las categorías.

\section{CONCLUSIÓN}

El resultado, por lo tanto, de este repaso necesariamente breve ha sido la constatación interesante de la presencia significativa en los objetivos de los PUMs en las universidades españolas de varias de las características del ocio humanista que previamente habíamos señalado como las más representativas tomando como referencia tanto la trayectoria investigadora del Instituto de Estudios de Ocio de la Universidad de Deusto como la aplicación directa en los programas de esta misma institución.

Si bien dicha presencia se debe rastrear en muchos casos de manera implícita o bien haciendo una interpretación flexible de las categorías, partimos del hecho de que las universidades estudiadas no tienen como horizonte de sus propuestas la investigación explícita sobre el ocio. Por ello, lo que buscábamos al aplicar la sencilla comparación que hemos llevado a cabo no era señalar ningún tipo de deficiencia; bien al contrario, debemos concluir que, pese a no compartir de antemano los conceptos señalados, hay interesantes coincidencias en el desarrollo de los objetivos de muchos de estos programas. 
Principios educativos ocio humanista en los... | M..$\underline{a}$ Luisa Amigo Fernández de Arroyabe et al.

Esto permite extraer como consecuencias tanto la solidez de las líneas generales que las universidades españolas están aplicando en el desarrollo de la formación permanente como el interés que puede derivarse de continuar esta línea de investigación acerca de los valores del ocio humanista como base para la propuesta de los PUMs ampliando su alcance respecto a las pretensiones de este breve artículo; primero, como contribución al interés investigador en el marco de la AEPUM, dado que, como hemos comprobado al inicio de este artículo, esta idea no se encuentra hasta el momento entre sus investigaciones destacadas; segundo, por el propio interés de ofrecer un apoyo teórico que pueda contribuir a enriquecer los planteamientos a partir de una comprensión más explícita de los principios del ocio. En este sentido, sería de especial interés desarrollar aquellas ideas que se han encontrado menos definidas en el estudio anterior, especialmente reforzar la importancia de la satisfacción como meta de la formación en los adultos y precisar el concepto de autotelismo para favorecer su comprensión más ajustada.

En definitiva, se puede afirmar que el análisis de los programas educativos para mayores, estudiados aquí, comparten con la teoría de ocio humanista unos principios educativos fundamentales, que pueden condensarse en las ideas de: experiencia, libertad, personalización y valores.

\section{REFERENCIAS BIBLIOGRÁFICAS}

Amigo, M. ${ }^{a}$ L. (2014). Ocio estético valioso. Documentos de Estudios de Ocio, n. ${ }^{\circ}$ 53. Bilbao: Universidad de Deusto.

Amigo, M. ${ }^{a}$ L. y Cuenca Amigo, M. (2012). Propuesta de líneas de mejora de la experiencia operística desde el ocio creativo. Arbor Ciencia, Pensamiento y Cultura, n. ${ }^{\circ} 188$, pp. 427-440.

Bru, C. (2002). Los Modelos Marco de los Programas Universitarios para Mayores. Alicante: Consellería de Bienestar Social.

Cabedo, S. y Alfageme, A. (2006). Los Programas Universitarios para Mayores en España: Una investigación sociológica. Castellón de la Plana: Publicaciones de la Universidad Jaume I.

Caride, J.A. (2012). Lo que el tiempo educa: el ocio como construcción pedagógica y social. Arbor Ciencia, Pensamiento y Cultura, n. ${ }^{\circ}$ 188, pp. 301-313.

Cicerón, M.T. (1994). Discurso en defensa de Publio Sestio, 96. En Discursos IV. Madrid: Gredos.

Csikszentmihalyi, I.S. (1998). Experiencia óptima. Estudios psicológicos del flujo de la conciencia. Bilbao: Desclée De Brouwer. 
Principios educativos ocio humanista en los... | M..$\underline{a}$ Luisa Amigo Fernández de Arroyabe et al.

Cuenca Amigo, J. (2012). El valor de la experiencia de ocio en la modernidad tardía. Colección de Documentos de Estudios de Ocio, n. ${ }^{\circ}$ 48. Bilbao: Universidad de Deusto.

Cuenca, M. (2000). Ocio humanista. Dimensiones y manifestaciones actuales del Ocio. Colección de Documentos de Estudios de Ocio, n. ${ }^{\circ}$ 16. Bilbao: Universidad de Deusto.

Cuenca, M. (2004). Pedagogía del Ocio: Modelos y propuestas. Bilbao: Universidad de Deusto.

Cuenca, M. (2005). Ocio solidario. La experiencia en grupos de jóvenes y jubilados. Documentos de Estudios de Ocio, n. ${ }^{\circ} 29$. Bilbao: Universidad de Deusto.

Cuenca, M. (2009). La educación del ocio, una parte esencial de la educación. En LópezBarajas, E. (coord.). Estrategias de formación en el siglo XXI: LIFE LONG LEARNING. (pp. 199-221). Madrid: Ariel.

Cuenca, M. (2010). La fuerza transformadora del ocio e Innovar y aprender disfrutando. En Cuenca, M.; Aguilar, E. y Ortega, C. Ocio para innovar (pp. 17-140). Documentos de Estudios de Ocio, núm. 42. Bilbao: Universidad de Deusto.

Cuenca Cabeza, M. (2011). Valores que dimanan del ocio humanista. En Madariaga, A. y Cuenca, J. (eds.). Los valores del ocio: cambio, choque e innovación (pp. 17-47). Documentos de Estudios de Ocio, núm. 43. Bilbao: Universidad de Deusto.

Cuenca, M. (2014). Ocio valioso. Documentos de Estudios de Ocio, n. ${ }^{\circ}$ 52. Bilbao: Universidad de Deusto.

Cuenca, M.; Lázaro, Y.; Amigo, M. ${ }^{a}$ L, y Cuenca, J. (2012). Ocio experiencial en la universidad: el programa multigeneracional cultura y solidaridad. Memorialidades, $\mathrm{n} .{ }^{0} 17$ (enero-junio 2012), pp. 475- 510.

Gómez, I. y Martínez, S. (2002). Educación del ocio en la edad adulta: El Graduado Universitario. En De La Cruz Ayuso, C. (ed.). Educación el Ocio. Propuestas internacionales (pp. 103-118). Documentos de Estudios de Ocio, n. ${ }^{\circ}$ 23. Bilbao: Universidad de Deusto.

Faché, W. (2002). El aprendizaje como medio para disfrutar del ocio y el aprendizaje como fin del ocio. En De La Cruz, C. (ed.). Educación del Ocio. Propuestas internacionales (pp. 85-102). Documentos de Estudios de Ocio, n. ${ }^{\circ}$ 23. Bilbao: Universidad de Deusto.

Hutchinson, S. y Robertson, B. (2012). Educación para el Ocio: Ha llegado la hora de un nuevo objetivo para una vieja idea. SIPS - Pedagogía Social. Revista interuniversitaria, n. ${ }^{0}$ 19, pp. 127-139. DOI: http://dx.doi.org/10.7179/PSRI_2012.19.09

Kleiber, D. A. (2000). Implicaciones del compromiso y la separación como experiencias de ocio relativas al desarrollo humano. En Cuenca Cabeza, M. (ed.) Ocio y desarrollo 
Principios educativos ocio humanista en los... | M..$\underline{a}$ Luisa Amigo Fernández de Arroyabe et al.

humano. Propuestas para el $6{ }^{\circ}$ Congreso Mundial de Ocio (pp. 65-74). Bilbao: Universidad de Deusto.

Kleiber, D.A. (2002). La intervención para el desarrollo y la educación del ocio: Una perspectiva/visión a lo largo de la vida. En De La Cruz, C. (ed.). Educación del Ocio. Propuestas internacionales (pp. 69-83). Documentos de Estudios de Ocio, n. ${ }^{\circ}$ 23. Bilbao: Universidad de Deusto.

Kleiber, D.A. (2012). Optimizing leisure experience after 40. Arbor Ciencia, Pensamiento y Cultura, n. ${ }^{\circ} 188$, pp. 341-349.

Kleiber, D.A.; Walker, G.J. y Mannell, R.C. (2011). A social psychology of leisure (2. ${ }^{\mathrm{a}}$ ed.). State College: Venture Publishing.

Lázaro, Y. (2004). Equiparación de Oportunidades, No Discriminación y Derecho al Ocio. Boletín ADOZ, n. ${ }^{\circ}$ 28, pp. 33-38.

Lyubomirsky, S.; King, L. y Diener, E. (eds.). (2005). The Benefits of Frequent Positive Affect: Does Happiness Lead to Success? Psychological Bulletin, vol. 131(6), pp. 803855.

Monteagudo, M. J. y Cuenca, M. (2012). Los itinerarios de ocio desde la investigación: tendencias, retos y aportaciones. Pedagogía Social. Revista Interuniversitaria, n. ${ }^{\circ} 20$, pp. 103-135.

Nimrod, G. y Adoni, H. (2006). Leisure styles and life satisfaction among recent retirees in Israel. Aging and Society, n. ${ }^{\circ}$ 26, pp. 607-630.

Séneca, L. A. (1922). Sobre el ocio y De la brevedad de la vida. Traducción de S. Segura Munguía, a partir de la edición latina Dialogus, publicada en la Collection des Universités de France. Paris: Belles Lettres.

Silvestre, M. (2000). Los valores básicos de la sociedad. En Andrés, F.; Elzo, J. y otros. España 2000. Entre el localismo y la globalidad (pp. 25-47). Madrid: Fundación Santa María/Universidad de Deusto.

Stebbins, R.A. (1992). Amateurs, professionals and serious leisure. Montreal and Kingston: McGill-Queen’s University Press.

Stebbins, R.A. (2006). Extending the serious leisure perspective. En Elkington, S.; Jones, I. y Lawrence, L. Serious leisure: extensions and applications (pp. 1-4). Michigan: LSA.

Stebbins, R.A. (2008). Serious leisure: a perspective for our time. New Brunswick: Transaction. 


\section{ANEXO}

Tabla comparativa. Programas Universitarios para Mayores de las universidades españolas
E: EXPERIENCIA
L: LIBERTAD
S: SATISFACCIÓN ${ }^{2}$

A: AUTOTELISMO

P.V.: PERSONALIZACION Y VALORES ${ }^{3}$

\begin{tabular}{|c|c|c|c|c|c|}
\hline Universidad & E. & L. & S. & A. & P.V. \\
\hline Universidad de Santiago & $\mathrm{X}$ & $\mathrm{X}$ & & $\mathrm{X}$ & $\mathrm{X}$ \\
\hline Universidad de Vigo & $\mathrm{X}$ & $\mathrm{X}$ & & $\mathrm{X}$ & $\mathrm{X}$ \\
\hline Universidad de Islas Baleares & $\mathrm{X}$ & $\mathrm{X}$ & & $\mathrm{X}$ & $\mathrm{X}$ \\
\hline Universidad de La Laguna & $\mathrm{X}$ & $\mathrm{X}$ & & $\mathrm{X}$ & $\mathrm{X}$ \\
\hline Universidad de Alcalá & $\mathrm{X}$ & $\mathrm{X}$ & & $\mathrm{X}$ & $\mathrm{X}$ \\
\hline Universidad de Comillas & $\mathrm{X}$ & $\mathrm{X}$ & $\mathrm{X}$ & $\mathrm{X}$ & $\mathrm{X}$ \\
\hline Universidad de CEU & $\mathrm{X}$ & $\mathrm{X}$ & $\mathrm{X}$ & $\mathrm{X}$ & $\mathrm{X}$ \\
\hline Universidad Jaume I & $\mathrm{X}$ & $\mathrm{X}$ & & $\mathrm{X}$ & $\mathrm{X}$ \\
\hline Pontifica de Salamanca & $\mathrm{X}$ & $\mathrm{X}$ & & $\mathrm{X}$ & $\mathrm{X}$ \\
\hline Universidad Politécnica de Cartagena & $\mathrm{X}$ & & & & $\mathrm{X}$ \\
\hline Universidad Ramón LLull & $\mathrm{X}$ & $\mathrm{X}$ & & $\mathrm{X}$ & $\mathrm{X}$ \\
\hline Universidad de Salamanca & $\mathrm{X}$ & $\mathrm{X}$ & & $\mathrm{X}$ & $\mathrm{X}$ \\
\hline Universidad de Oviedo & $\mathrm{X}$ & $\mathrm{X}$ & $\mathrm{X}$ & $\mathrm{X}$ & $\mathrm{X}$ \\
\hline Universidad de Carlos III & $\mathrm{X}$ & & & & \\
\hline Universidad Complutense de Madrid & $\mathrm{X}$ & $\mathrm{X}$ & & $\mathrm{X}$ & $\mathrm{X}$ \\
\hline Universidad de Córdoba & $\mathrm{X}$ & & & $\mathrm{X}$ & $\mathrm{X}$ \\
\hline \multicolumn{6}{|l|}{ Universidad Politécnica de Valencia ${ }^{2}$} \\
\hline $\begin{array}{l}\text { Universidad José Saramago } \\
\text { (Castilla-La Mancha) }\end{array}$ & $\mathrm{X}$ & $\mathrm{X}$ & $\mathrm{X}$ & $\mathrm{X}$ & $\mathrm{X}$ \\
\hline \multicolumn{6}{|l|}{ Universidad Pública de Navarra ${ }^{3}$} \\
\hline Universidad de Extremadura & $\mathrm{X}$ & & & $\mathrm{X}$ & $\mathrm{X}$ \\
\hline Universidad de Murcia & $\mathrm{X}$ & $\mathrm{X}$ & & $\mathrm{X}$ & $\mathrm{X}$ \\
\hline UNED senior & $\mathrm{X}$ & $\mathrm{X}$ & & $\mathrm{X}$ & $\mathrm{X}$ \\
\hline \multicolumn{6}{|l|}{ Universidad de Huelva } \\
\hline Universidad de Almería & $\mathrm{X}$ & $\mathrm{X}$ & & & $\mathrm{X}$ \\
\hline Universidad de Granada & $\mathrm{X}$ & $\mathrm{X}$ & & $\mathrm{X}$ & $\mathrm{X}$ \\
\hline Universidad de A Coruña & $\mathrm{X}$ & $\mathrm{X}$ & & $\mathrm{X}$ & $\mathrm{X}$ \\
\hline
\end{tabular}

2 Escasa información en Web.

3 Escasa información en Web. 
INGURUAK [61] | 2016 | 23-42

Principios educativos ocio humanista en los... | M. $\underline{a}$ Luisa Amigo Fernández de Arroyabe et al.

\begin{tabular}{|l|c|c|c|c|c|}
\hline \multicolumn{1}{|c|}{ Universidad } & E. & L. & S. & A. & P.V. \\
\hline Universidad de Alicante & $\mathrm{X}$ & $\mathrm{X}$ & & $\mathrm{X}$ & $\mathrm{X}$ \\
\hline Universidad de Sevilla & $\mathrm{X}$ & $\mathrm{X}$ & & $\mathrm{X}$ & $\mathrm{X}$ \\
\hline Universidad de Valencia & $\mathrm{X}$ & $\mathrm{X}$ & & $\mathrm{X}$ & $\mathrm{X}$ \\
\hline Universidad de Málaga & $\mathrm{X}$ & $\mathrm{X}$ & & & \\
\hline Universidad de Rovira y Virgili & $\mathrm{X}$ & & & & \\
\hline Universidad de Barcelona & $\mathrm{X}$ & $\mathrm{X}$ & & $\mathrm{X}$ & $\mathrm{X}$ \\
\hline Universidad de Burgos & $\mathrm{X}$ & $\mathrm{X}$ & & $\mathrm{X}$ & $\mathrm{X}$ \\
\hline Universidad de Lleida & $\mathrm{X}$ & $\mathrm{X}$ & & & $\mathrm{X}$ \\
\hline Universidad de Valladolid & $\mathrm{X}$ & $\mathrm{X}$ & & $\mathrm{X}$ & $\mathrm{X}$ \\
\hline
\end{tabular}

